\title{
Análise do perfil facial masculino adulto jovem, esteticamente agradável, em fotografias padronizadas: comparação da medição manual com a computadorizada*
}

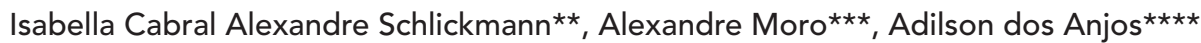

\section{Resumo}

Objetivo: analisar o perfil facial masculino adulto jovem, através de fotografias padronizadas e comparar a medição manual com a medição computadorizada por meio do software Cef X (CDT). Métodos: a amostra consistiu de 40 indivíduos brasileiros brancos, com média de idade de 21,9 anos, que possuíam perfis agradáveis, oclusão normal, sem histórico de tratamento ortodôntico prévio. As fotografias coloridas de $10 \mathrm{~cm} \times 15 \mathrm{~cm}$ foram obtidas de forma padronizada, com o indivíduo na posição natural da cabeça. As fotografias foram medidas manualmente, utilizando-se paquímetro digital e transferidor, e também através de computador, utilizando-se o programa Cef X (CDT). Trinta e seis variáveis faciais foram analisadas por meio de medidas lineares, angulares e proporcionais. Resultados: as médias da medição manual e da medição por computador foram diferentes estatisticamente para 24 variáveis. No método por computador, somente uma medida mostrou diferença significativa entre a primeira e a segunda medição, enquanto na manual oito mostraram diferença. Conclusões: considerando-se que houve diferença entre as medições manual e por computador, deve-se utilizar as médias respectivas para cada tipo de medição. Os dois tipos de medição são confiáveis, entretanto a medição por computador é mais precisa. Devido à praticidade e agilidade da medição por computador, esta seria a mais indicada.

Palavras-chave: Análise facial. Perfil facial. Ortodontia. Fotografias padronizadas.

\section{INTRODUÇÃO}

A avaliação da morfologia craniofacial é uma ferramenta indispensável na prática clínica e na pesquisa, e pode ser obtida por diferentes meios. Um destes meios é a fotografia, que não é cara, não expõe o paciente a uma radiação potencialmente nociva e pode proporcionar uma boa avaliação da harmonia dos relacionamentos entre estruturas craniofaciais externas, incluindo a contribuição dos tecidos moles ${ }^{10,15,16}$.

Uma vez que as técnicas sejam padronizadas, análises fotográficas podem ser um útil comple-

* Resumo da monografia apresentada ao curso de especialização em Ortodontia e Ortopedia Facial da EAP-ABO, Curitiba/PR.

** Especialista em Ortodontia e Ortopedia Facial pela EAP-ABO, Curitiba / PR.

*** Professor adjunto da UFPR - graduação e pós-graduação em Ortodontia. Professor titular da Universidade Positivo - graduação e pós-graduação em Ortodontia.

**** Mestre em Estatística e Experimentação Agronômica pela USP. Professor assistente do departamento de Estatística da UFPR. 
mento às análises radiográficas convencionais, acrescentando o fato de que, como toda ciência, a Ortodontia também necessita do auxílio de dados numéricos para exprimir o conceito de beleza ${ }^{10,12}$.

A utilização de análises cefalométricas confeccionadas em computador está amplamente difundida na Ortodontia. O mesmo não pode se dizer sobre a análise fotográfica. Apesar das fotografias faciais constarem de forma rotineira nas documentações ortodônticas realizadas no início do tratamento, elas têm sido exploradas mais de forma subjetiva, por meio de avaliações morfológicas ${ }^{11}$. Entretanto, em algumas situações clínicas, como no planejamento de casos para cirurgia ortognática, a obtenção de uma análise facial numérica é muito importante $\mathrm{1}^{10,12}$.

Este trabalho procura contribuir com esse tema, ao analisar o perfil facial masculino adulto jovem através de fotografias padronizadas e comparar a medição manual com a medição computadorizada por meio do software Cef X (CDT).

\section{MATERIAL}

A amostra estudada foi constituída de estudantes do gênero masculino, dos cursos de Odontologia e Medicina da Universidade Federal de Santa Catarina.

Trezentos estudantes foram entrevistados e examinados individualmente, com a finalidade de se verificar as seguintes condições: serem leucodermas, filhos diretos de pais brasileiros e de descendência européia (portuguesa, italiana, espanhola ou alemã); não deveriam ter recebido tratamento ortodôntico de qualquer espécie; não podiam ter sido submetidos a cirurgia plástica facial e tinham que apresentar relação molar de Classe I, sendo aceitos pequenos apinhamentos e diastemas. $\mathrm{O}$ exame dentário foi realizado com o auxílio de duas espátulas descartáveis de madeira.

Nesta etapa, foi obtida uma amostra de 67 rapazes, que foram fotografados de perfil, de forma padronizada.

Para a realização das fotografias, foram utiliza- dos: uma máquina Canon T60, lente Canon Macro Lens FD 100mm, flash circular Vivitar Macroflash 5000, tripé Vivitar VPT-10, filmes Kodak Gold coloridos ASA 100/35mm, uma linha azul com um peso em sua extremidade, para dar a referência vertical, e um espelho de $48 \mathrm{~cm}$ x $88 \mathrm{~cm}$.

\section{MÉTODOS \\ Fotografias}

A máquina fotográfica foi posicionada sobre o tripé a um metro (indicado pela regulagem da lente macro $100 / 35 \mathrm{~mm}$ ) da face do mesmo e o pesquisador ajustava a câmera até que o indivíduo ficasse claramente em foco, indicando que a distância desejada tinha sido obtida ${ }^{10,13}$.

$\mathrm{O}$ indivíduo ficava sentado em uma cadeira, tendo na parede do seu lado esquerdo, um pouco à frente da sua face, uma linha azul presa por uma fita adesiva e com um peso em sua extremidade inferior, para dar a relação com o solo, que seria a linha vertical verdadeira ${ }^{5}$. Para auxiliar os indivíduos, um espelho foi colocado à cerca de um metro à frente da cadeira, e estes eram orientados a sentarem retos nesta cadeira e olharem para o reflexo de suas pupilas no espelho, com a finalidade de centralizá-las nos olhos. Caso o pesquisador observasse que o indivíduo encontrava-se em posição inadequada, ele o orientava para que assumisse a postura correta. Desta forma, todos foram fotografados na posição natural da cabeça $^{17}$. As fotografias foram realizadas sempre pelo mesmo operador e o perfil de cada indivíduo foi fotografado duas vezes. A primeira com os dentes em posição de repouso e a segunda em máxima intercuspidação habitual, sendo que em ambas as fotografias os lábios ficavam levemente selados e relaxados.

Todos os filmes foram revelados num mesmo laboratório fotográfico Kodak com padrão QLAB (Padrão de Qualidade Internacional Kodak). Estas fotografias foram impressas sobre papel Kodak, de acordo com as técnicas de processamento e desenvolvimento padrão, e obteve-se fotografias colori- 
das de $10 \mathrm{~cm} \times 15 \mathrm{~cm}$.

Para a aferição da distorção entre o tamanho real da face e o tamanho da fotografia, 10 indivíduos fotografados tiveram dois pontos marcados em sua face, com uma distância de $1 \mathrm{~cm}$ entre eles, na região da bochecha. A distância entre estes pontos na fotografia foi medida três vezes para cada um dos dez indivíduos, a mediana foi calculada e destas medianas foi obtida uma média de $5,57 \mathrm{~mm}$. Cada $1 \mathrm{~cm}$ real na face do indivíduo equivalia a $0,557 \mathrm{~cm}$ nas fotografias. Para saber o valor real da medida, foi aplicado um valor de correção sobre as medidas, que foi obtido através de uma regra de três simples, onde foi constatado que $1 \mathrm{~cm}$ na foto equivalia a $1,7953 \mathrm{~cm}$ no indivíduo real.

\section{Seleção da amostra final}

Esta amostra de 67 indivíduos foi submetida a uma banca, composta por 12 alunos que estavam concluindo o curso de pós-graduação em Ortodontia da ABO-PR, que individualmente tiveram acesso às fotografias, as analisaram e assinalaram em uma ficha, dentre estes perfis, os que julgaram agradáveis e os não-agradáveis.

Foi feito um levantamento das 12 fichas de seleção da banca dos 12 alunos, sendo que os perfis que vieram a fazer parte da amostra final foram os que obtiveram $50 \%$ ou mais de aceitação nestas fichas. Assim, chegou-se a uma amostra final de 40 indivíduos, com média de idade de 21,9 anos (variando de 17,4 a 32,1 anos).

\section{Medição manual}

O traçado fotográfico foi realizado com uma lapiseira (Pentel P203), grafite 0,3mm (Pentel 3H), régua (Desetec mod 7120) e esquadro (Desetec mod $2521-45^{\circ}$ ), em papel Ultraphan de acetato (Grafix), que foi fixado à fotografia com fita adesiva. As medidas angulares foram obtidas com transferidor com precisão de $0,5^{\circ}$ (Desetec mod $8115-180^{\circ}$ ), e as lineares com paquímetro eletrônico digital, de resolução de $0,01 \mathrm{~mm}$, exatidão de $0,03 \mathrm{~mm}$ e repetibilidade de $0,01 \mathrm{~mm}$ (Starret $\mathrm{n}^{\circ}$ cat $727 \mathrm{ME}-6 / 150 \mathrm{~mm}$ ).

As medidas foram registradas em uma ficha para cada indivíduo, num total de 40 fichas. Destes 40 indivíduos, 15 foram selecionados aleatoriamente e, entre 7 e 15 dias da primeira medição, foram retraçados e remedidos e os valores registrados em outra ficha, com a finalidade de verificar se havia ou não diferenças nestas medidas.

\section{Medição por computador}

As fotografias foram escaneadas (Scanner Scan Jet 4c/T HP Hewlett Packard) com 150 dpi, formato JPG e colocadas no programa Cef X Cefalometria Computadorizada - CDT (Cuiabá / MT), que foi adequadamente individualizado para esta pesquisa pela empresa CDT, para possuir todas as medidas utilizadas na medição manual utilizada e também realizá-las exatamente com a mesma metodologia.

Cada paciente foi cadastrado e sua imagem importada para o programa. Antes da marcação dos pontos, foi definido o ângulo de cada fotografia e esta foi angulada para que a linha vertical verdadeira ficasse em sua posição correta. Após esta etapa, os pontos foram marcados e o relatório de medidas impresso.

Nas medições por computador, os mesmos 15 indivíduos remedidos manualmente também tiveram seus pontos marcados novamente e seus relatórios de medidas reimpressos, entre 7 e 15 dias da primeira medição, para verificar a confiabilidade do método.

\section{Traçado fotográfico}

Os pontos, as linhas e as medidas fotométricas utilizados, descritos no trabalho de Reche et al. ${ }^{10}$, são apresentados nas figuras 1 a 5 .

\section{Análise estatística}

A análise estatística foi dividida em medidas lineares e medidas angulares e foi realizada com os valores reais.

Para cada uma das variáveis lineares da amostra 


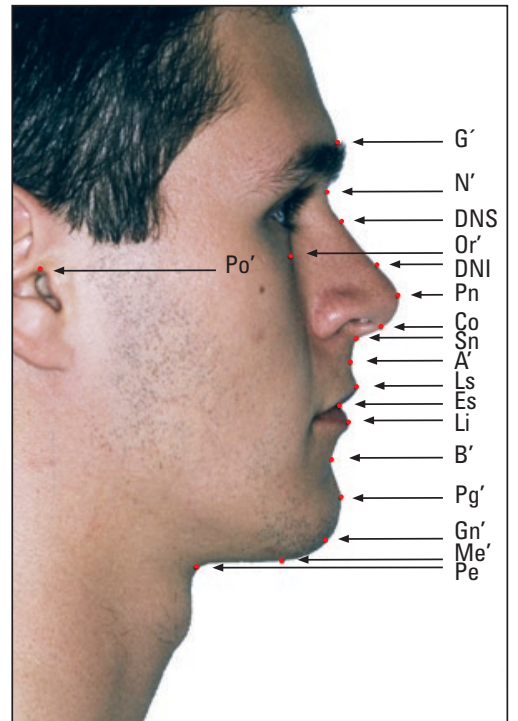

FIGURA 1 - Pontos fotográficos: G' (glabela); N' (násio); Pn (ponta do nariz); Co (columela); Sn (subnasal); A'; Ls (lábio superior); Es (estômio); Li (lábio inferior); B'; Pg' (pogônio); Gn' (gnátio); Me' (mentoniano); Pe (pescoço); Or' (orbitário); Po' (pório); DNS (dorso nasal superior); DNI (dorso nasal inferior).

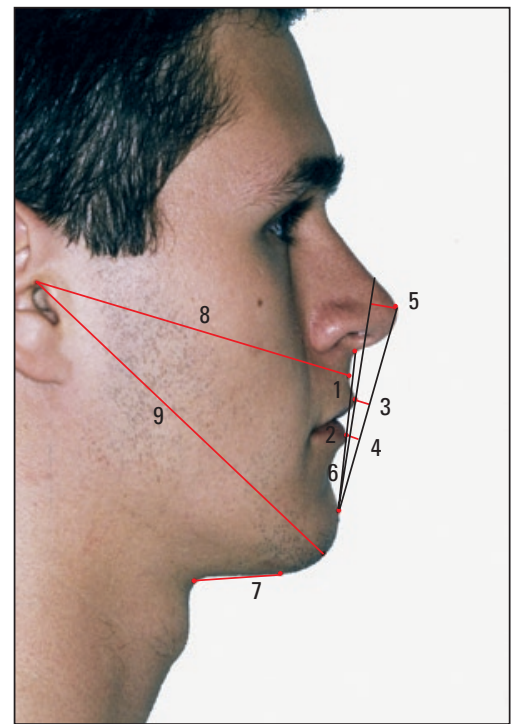

FIGURA 2 - Medidas lineares: (1) linha de Burstone $\left(\mathrm{Sn}-\mathrm{Pg}^{\prime}\right)$ ao $\mathrm{Ls}$; (2) linha de Burstone $\left(\mathrm{Sn}-\mathrm{Pg}^{\prime}\right)$ ao $\mathrm{Li}$; (3) linha de Ricketts $\left(\mathrm{Pn}-\mathrm{Pg}^{\prime}\right)$ ao Ls; (4) linha de Ricketts $\left(\mathrm{Pn}-\mathrm{Pg}^{\prime}\right)$ ao $\mathrm{Li}$; (5) linha de Holdaway (Pg'-Ls) ao $\mathrm{Pn}$; (6) linha de Holdaway (Pg'-Ls) a Li; (7) comprimento da linha mento-pescoço (Me'-Pe); (8) comprimento maxilar $\left(\mathrm{Po}^{\prime}-\mathrm{A}^{\prime}\right)$; (9) comprimento mandibular (Po'-Gn').

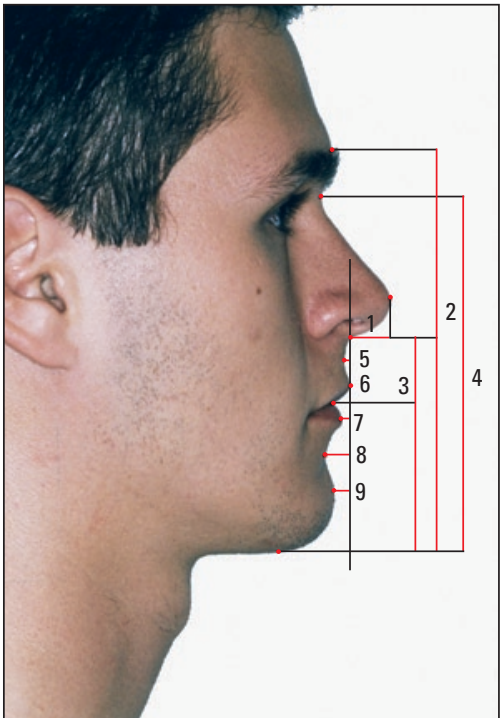

FIGURA 3 - Medidas lineares e proporcionais: (1) comprimento nasal (Sn-Pn); (2) proporção facial total (G'-Sn-Me'); (3) proporção do 1/3 inferior (SnEs-Me'); (4) altura facial ( $\left.\mathrm{Na}^{\prime}-\mathrm{Me}^{\prime}\right) ;$ (5) A' a LVV através do $S n ;$ (6) Ls a LVV através do Sn; (7) Li a LVV através do $\mathrm{Sn}$; (8) B' a LVV através do $\mathrm{Sn}$; (9) $\mathrm{Pg}^{\prime}$ a LVV através do Sn; (10) harmonia intermaxilares $A^{\prime}$ a B'.

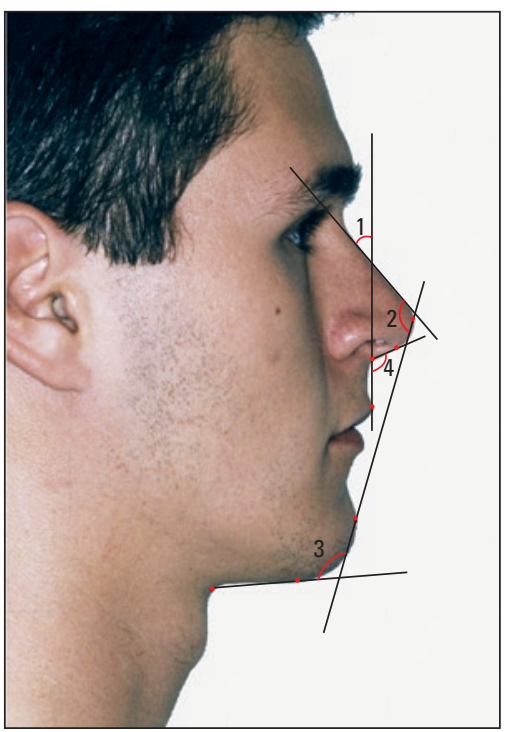

FIGURA 4 - Medidas angulares: (1) ângulo de Powell nasofacial (LVV através do Sn-linha tangente ao dorso do nariz); (2) ângulo de Powell nasomental (linha tangente ao dorso do nariz-linha de Ricketts); (3) ângulo de Powell mento-cervical (linha de Ricketts-linha mento-pescoço); (4) ângulo nasolabial (CoSn-Ls); (5) ângulo do lábio superior (LVV através do Sn-lábio superior Sn-Ls).

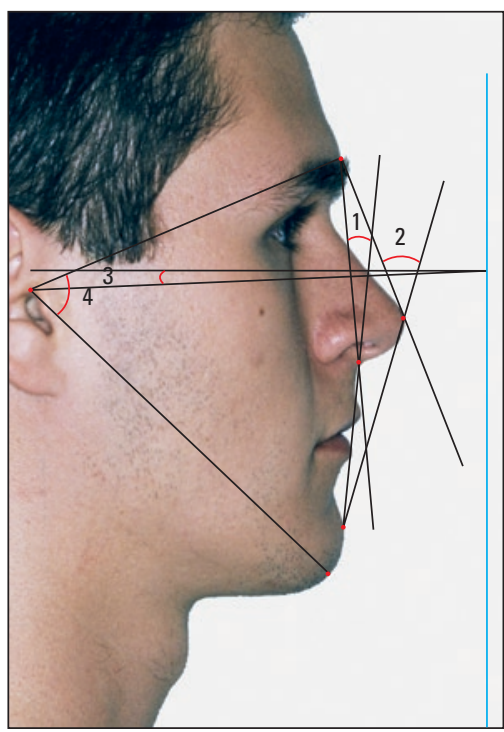

FIGURA 5 - Medidas angulares: (1) ângulo da convexidade facial (G'-Sn-Pg'); (2) ângulo da convexidade facial total $\left.\left(G^{\prime}-P n-P g\right)^{\prime}\right)$ (3) inclinação do plano de Frankfurt (Po'-Or'-horizontal verdadeira); (4) cone facial de Reche $\left(G^{\prime}-P 0^{\prime}-G n^{\prime}\right)$. 
analisadas, foram realizadas as seguintes estatísticas descritivas: média, desvio-padrão (d.p.), desviopadrão do erro (d.p.e.), mediana, valor mínimo e valor máximo. Para as medidas angulares, foram calculadas: média angular, desvio angular, mínimo, máximo, limites de confiança para a média populacional de cada variável e o desvio-padrão do erro (d.p.e.).

Objetivando testar a hipótese de que as variáveis lineares seguem uma distribuição normal, foi efetuado o teste de Lilliefors ${ }^{4}$, considerando-se um nível de significância de 5\%.

Para constatar se havia diferença entre as medidas (altura Sn-Me' e altura facial) em repouso e em oclusão, foi aplicado o teste $t$ pareado para amostras dependentes, quando as medidas apresentavam normalidade. Foi usado o teste de Wilcoxon para aquelas que não apresentavam normalidade, considerando-se um nível de significância de $5 \%$.

Para constatar essa mesma diferença entre repouso e oclusão das medidas angulares (inclinação do plano de Frankfurt e cone facial), foi aplicado o teste de Moore ${ }^{18}$ com um nível de $5 \%$ entre as médias.

Com o objetivo de testar a confiabilidade das medidas, foi aplicado o teste de Lilliefors ${ }^{4}$ nas médias da primeira e segunda medição e para aquelas variáveis lineares que apresentaram normalidade foi efetuado o teste t pareado para amostras dependentes, com o objetivo de testar o erro sistemático. Para aquelas que não apresentaram normalidade foi usado o teste de Wilcoxon, considerando-se um nível de significância de 5\%.

Com o objetivo de avaliar o erro casual, foi calculado o desvio-padrão do erro utilizando-se a fórmula de Dahlberg ${ }^{8} \mathrm{DP}_{\mathrm{E}}=\left(\Sigma \mathrm{D}^{2} / 2 \mathrm{~N}\right)^{1 / 2}$. Quando estes foram superiores a $1 \mathrm{~mm}$ para as medidas lineares, foram considerados erros significantes ${ }^{9}$.

Como para as medidas lineares, para testar o erro sistemático, e assim a confiabilidade das medidas angulares, foram calculadas as médias da primeira $(n=15)$ e da segunda $(n=15)$ medição, medição manual e por computador. Então, para aferir se havia diferença entre os valores das médias da primeira e da segunda medição, foi utilizado o teste de Moore ${ }^{18}$.

O erro casual também foi calculado com a fórmula de Dahlberg ${ }^{8}$. Porém, os valores dos desviospadrão do erro foram considerados erros significantes somente quando superiores a 1,5 $\mathrm{grau}^{9}$.

Para testar se havia diferença significativa $(\mathrm{p}<$ $0,05)$ entre as médias medidas manualmente e as aferidas por computador nas fotografias realizadas em repouso, utilizou-se o teste $t$ para dados pareados. Utilizou-se o teste de Wilcoxon para as variáveis que não apresentaram distribuição normal e o teste de Moore para as variáveis angulares, todos com um nível de significância de 5\%.

\section{RESULTADOS}

Após a tabulação dos dados, chegou-se aos resultados que são apresentados nas tabelas 1 a 3 .

As tabelas mostram que, das 36 variáveis aferidas, somente 12 tiveram as suas médias da medição manual e da medição por computador sem diferenças estatisticamente significantes. Contudo, nas 24 variáveis com diferenças estatisticamente significantes, a diferença entre as médias foi de $0,2 \mathrm{~mm}$ a $1,5 \mathrm{~mm}$ e de $0,4^{\circ}$ a $1,2^{\circ}$.

O teste $t$, usado para detectar erros sistemáti$\cos ^{8}$ da medição manual, mostrou que - para as variáveis distância do lábio inferior em relação à linha de Burstone, distância do lábio superior em relação à linha de Ricketts, comprimento da linha mento-pescoço, comprimento maxilar e comprimento mandibular - existiu diferença estatisticamente significativa $(\mathrm{p}<0,05)$, entre a primeira e a segunda medição, ou seja, estas medidas não foram consideradas estatisticamente confiáveis. $\mathrm{O}$ comprimento da linha mento-pescoço obteve a maior diferença numérica entre as médias: primeira medição $38 \mathrm{~mm}$ e segunda medição $37 \mathrm{~mm}$.

De acordo com o teste de Wilcoxon da medição manual, somente para a variável distância do lábio superior em relação à linha de Burstone 
Tabela 1 - Comparação entre as medidas lineares realizadas manualmente e com computador, por meio do teste t pareado $(n=40)$, nas fotografias em repouso.

\begin{tabular}{|c|c|c|c|c|c|}
\hline \multirow{2}{*}{ variáveis } & \multicolumn{2}{|c|}{ manual } & \multicolumn{2}{|c|}{ computador } & \multirow[b]{2}{*}{$p$-valor $(p<0,05)$} \\
\hline & média (mm) & d.p. & média (mm) & d.p. & \\
\hline linha de Burstone Ls & 3,2 & 1,5 & 2,8 & 1,5 & 0,00000 \\
\hline linha de Ricketts Ls & $-4,9$ & 1,7 & $-4,6$ & 1,8 & 0,00014 \\
\hline linha de Ricketts Li & $-2,8$ & 2,3 & $-2,6$ & 2,3 & 0,10439 \\
\hline linha de Holdaway Pn & 8,5 & 3,1 & 8,3 & 3,1 & 0,01052 \\
\hline linha de Holdaway Li & 0,4 & 1,5 & 0,3 & 1,5 & 0,07236 \\
\hline comprimento nasal & 14,8 & 2,0 & 14,3 & 1,9 & 0,00000 \\
\hline comprimento mandibular & 134,5 & 7,2 & 133,0 & 7,0 & 0,00000 \\
\hline proporção facial total & 0,9 & 0,1 & 0,9 & 0,1 & 0,14394 \\
\hline proporção do $1 / 3$ inferior & 0,5 & 0,0 & 0,5 & 0,0 & 0,03315 \\
\hline altura Sn-Me' oclusão & 71,1 & 5,0 & 70,3 & 5,3 & 0,00000 \\
\hline altura Sn-Es & 23,4 & 2,3 & 23,3 & 2,4 & 0,20602 \\
\hline paralela a LVV por Sn ao Li & $-0,4$ & 2,4 & $-0,7$ & 2,3 & 0,00000 \\
\hline paralela a LVV por Sn ao B' & $-7,3$ & 2,9 & $-7,4$ & 2,9 & 0,33302 \\
\hline altura facial repouso & 123,0 & 6,7 & 122,4 & 7,1 & 0,00308 \\
\hline altura facial oclusão & 121,6 & 6,8 & 121,0 & 6,8 & 0,00003 \\
\hline
\end{tabular}

Tabela 2 - Comparação entre as medidas lineares realizadas manualmente e por com computador por meio do teste de Wilcoxon $(n=40)$, nas fotografias em repouso.

\begin{tabular}{|c|c|c|c|c|c|}
\hline \multirow{2}{*}{ variáveis } & \multicolumn{2}{|c|}{ manual } & \multicolumn{2}{|c|}{ computador } & \multirow[b]{2}{*}{$\mathrm{p}$-valor $(\mathrm{p}<0,05)$} \\
\hline & média (mm) & d.p. & média (mm) & d.p. & \\
\hline linha de Burstone Li & 2,3 & 2,0 & 2,0 & 1,9 & 0,000002 \\
\hline comprimento mento-pescoço & 35,9 & 5,2 & 36,3 & 5,4 & 0,146606 \\
\hline comprimento maxilar & 115,1 & 6,7 & 114,0 & 6,4 & 0,000000 \\
\hline altura G'-Sn & 65,4 & 4,3 & 65,4 & 3,9 & 0,726736 \\
\hline altura $\mathrm{Sn}-\mathrm{Me}^{\prime}$ & 72,5 & 5,1 & 71,7 & 5,2 & 0,000026 \\
\hline altura Es-Me' & 49,2 & 3,5 & 48,4 & 3,6 & 0,000000 \\
\hline paralela a LVV por Sn ao Ls & 1,8 & 1,8 & 1,4 & 1,8 & 0,000000 \\
\hline paralela a LVV por Sn ao $\mathrm{Pg}$ & $-4,6$ & 3,8 & $-4,8$ & 3,7 & 0,034859 \\
\hline paralela a LVV por Sn ao A' & $-0,9$ & 0,8 & $-1,0$ & 0,9 & 0,419606 \\
\hline harmonia intermaxilares $A^{\prime}$ a $B^{\prime}$ & 6,5 & 2,6 & 6,4 & 2,5 & 0,162154 \\
\hline
\end{tabular}


Tabela 3 - Comparação entre as medidas angulares realizadas manualmente e por com computador por meio do teste de Moore $(n=40)$, nas fotografias em repouso.

\begin{tabular}{|c|c|c|c|c|c|c|}
\hline \multirow[b]{2}{*}{ variáveis } & \multicolumn{2}{|c|}{ manual } & \multicolumn{2}{|c|}{ computador } & \multirow[b]{2}{*}{$\mathrm{R}^{\prime} \alpha, 1$} & \multirow[b]{2}{*}{$\mathrm{R}^{\prime}$} \\
\hline & média angular $\left({ }^{\circ}\right)$ & desvio angular & média angular $\left(^{\circ}\right)$ & desvio angular $\left({ }^{\circ}\right)$ & & \\
\hline âng. Powell nasofacial & 38,0 & 4,7 & 39,0 & 4,2 & 1,012 & 1,850 \\
\hline âng. Powell nasomental & 126,0 & 4,3 & 125,0 & 4,1 & 1,012 & 1,601 \\
\hline âng. Powell mento-cervical & 120,0 & 6,9 & 120,2 & 6,8 & 1,012 & 0,363 \\
\hline ângulo nasolabial & 107,0 & 9,6 & 108,0 & 9,3 & 1,012 & 1,468 \\
\hline convexidade facial & 12,1 & 4,1 & 12,2 & 3,9 & 1,012 & 0,181 \\
\hline convexidade facial total & 39,3 & 3,7 & 38,8 & 3,9 & 1,012 & 1,914 \\
\hline inclinação de Frankfurt & 6,6 & 4,5 & 6,1 & 4,6 & 1,012 & 2,044 \\
\hline cone facial & 62,7 & 3,0 & 63,1 & 2,9 & 1,012 & 1,220 \\
\hline ângulo do lábio superior & 5,8 & 5,9 & 4,6 & 5,8 & 1,012 & 2,338 \\
\hline
\end{tabular}

existiu diferença estatisticamente significante $(\mathrm{p}<$ $0,05)$ entre a primeira e a segunda medição e novamente a diferença foi mínima. Assim, esta medida não foi considerada estatisticamente confiável.

Somente os ângulos de Powell nasomental e mentocervical da medição manual monstraram diferença entre a primeira e a segunda medição, com um nível de significância de 5\%. Para o ângulo de Powell nasomental, as médias angulares da primeira e da segunda medição foram, respectivamente, $126,5^{\circ}$ e $125,6^{\circ}$. Vemos que essa diferença entre as médias foi pequena, cerca de $1^{\circ}$. Já para o ângulo mentocervical, ambas as médias foram $119,1^{\circ}$, mostrando a inexistência de uma diferença numérica em apenas uma casa após a vírgula, mas com diferença estatisticamente significante.

Verificando os erros casuais na medição manual, somente a medida linear comprimento da linha mento-pescoço apresentou o desvio-padrão do erro $^{8} \mathrm{com}$ valor superior a $1 \mathrm{~mm}$, mostrando que esta medida apresentou um erro casual significativo, provavelmente devido à dificuldade na localização precisa dos pontos envolvidos na medida (os pontos Me' e Pe).
Só o ângulo nasolabial, dentre as medidas angulares, apresentou valor acima de $1,5^{\circ}$ para o desvio-padrão do erro.

Para a medição por computador, ao se comparar as médias da primeira medição com as da segunda, somente a variável distância do lábio superior em relação à linha de Burstone mostrou um p-valor de 0,015912, apresentando uma diferença estatisticamente significante entre a primeira e a segunda medição. Novamente, o comprimento da linha mento-pescoço e o ângulo nasolabial foram as únicas medidas da medição por computador a apresentarem os valores do desvio-padrão do erro ${ }^{8}$ significativos.

\section{DISCUSSÃO}

No presente trabalho, constatou-se que, tanto na medição manual quanto na medição por computador, há uma diferença altamente significativa entre a medida na fotografia em repouso e em oclusão para as medidas altura facial, altura SnMe' e cone facial. Por isso, deve-se ter o cuidado de estabelecer em todas as fotos a mesma posição, para permitir a padronização e a comparação. 
Para a medida inclinação do plano de Frankfurt, não houve diferença entre a fotografia em repouso e em oclusão e, tendo em vista que o paciente não permaneceu parado entre uma fotografia e outra, pode-se, então, afirmar que dentro deste curto espaço de tempo, entre a primeira e a segunda fotografia, a posição natural da cabeça foi reproduzivel, tanto na medição manual quanto na por computador.

Ao comparar os erros casuais encontrados nesse estudo com outros, observa-se que Reche et al. ${ }^{10}$ também encontraram que a única medida linear a apresentar o desvio-padrão do erro superior a $1 \mathrm{~mm}$ foi a linha mento-pescoço (com o valor de $1,5 \mathrm{~mm}$ ) e a única angular superior a $1,5^{\circ}$ foi o ângulo nasolabial (com 2, $6^{\circ}$ ), reafirmando a dificuldade encontrada na tomada destas duas medidas.

Segundo Arnett e Bergman ${ }^{1}$, o comprimento da linha mento-pescoço não necessita de mensuração milimétrica. Para Suguino et al. ${ }^{14}$, o comprimento desta linha deveria ser de, aproximadamente, $40 \mathrm{~mm}$. Nesta amostra, obteve-se $35,9 \mathrm{~mm}$ na medição manual e $36,3 \mathrm{~mm}$ na por computador. Porém, esta medida não se mostrou confiável nos dois meios de medição tendo, para as duas, valores superiores a $1 \mathrm{~mm}(1,2 \mathrm{~mm}$ e $1,6 \mathrm{~mm})$ para o desvio-padrão do erro. Talvez tenha ocorrido um erro, devido à dificuldade na tomada desta medi$\mathrm{da}$, pois o valor encontrado foi menor que o encontrado por Reche et al. ${ }^{10}-38 \mathrm{~mm}$ num grupo do gênero feminino, que também apresentou um valor significativo do desvio-padrão do erro. Esses dados sugerem que essa medida deve ser utilizada com restrições.

Os ângulos de Powell nasofacial e nasomental na medição manual tiveram altos valores do desvio-padrão do erro $\left(1,3^{\circ}\right)$, mas não significativos. Provavelmente devido à linha do dorso do nariz, que faz parte dos dois ângulos em questão, e foi traçada como uma linha tangente ao dorso do nariz, muito subjetivamente neste tipo de medição. Na medição por computador, estes ângulos tiveram valores do desvio-padrão do erro bem baixos $\left(0,4^{\circ}\right)$, talvez devido ao fato de que o computador para traçar uma linha precisa de dois pontos. Então, criou-se os pontos dorso nasal superior (DNS) e dorso nasal inferior (DNI). Marcava-se os dois pontos e o computador traçava a linha, provavelmente de maneira bem mais precisa do que na medição manual. Sugere-se, então, a utilização destes pontos também para a medição manual.

Em muitas das medidas que apresentaram diferenças está presente o ponto subnasal, que segundo Bishara et al. ${ }^{2}$ é um ponto de referência pouco confiável. Cummins, Bishara e Jakobsen ${ }^{6}$ afirmaram que medidas que incluem os pontos subnasal, pogônio' e gnátio' tendem a ser menos confiáveis. Strauss et al. ${ }^{13}$ constataram que há uma dificuldade na reprodutibilidade da localização do mento de tecido mole.

Neste trabalho, foram cometidos erros sistemáticos pelo fato de ter havido diferenças no período de tempo, pois a prática do observador mudou com a experiência, isto é, a primeira medição manual foi quase sem experiência, na segunda manual esta já aumentou um pouco, na primeira por computador mais um pouco e na segunda por computador ainda mais, registrando o fato de que, por problemas técnicos, o período de tempo entre a medição manual e a por computador foi de cerca de 6 meses. Então, uma série de medidas pode diferir sistematicamente de uma série feita num tempo diferente. Isto pode ter ocorrido entre a medição manual e a medição por computador, o que pode ter contribuído para as várias diferenças significativas entre estas médias ${ }^{8}$.

Importante salientar que, na medição por computador, somente uma medida mostrou diferença estatisticamente significativa entre a primeira e a segunda medição, e foi encontrado desvio-padrão do erro significativo para somente outras duas variáveis. Na medição manual, as mesmas duas variáveis da medição por computador apresentaram valores do desvio-padrão do erro significativos e, destas, uma e mais sete variáveis (num total de oito variáveis) mostraram diferença significativa 
entre as médias da primeira e da segunda medição. Este fato da medição por computador ter se mostrado mais confiável, pode ser devido a esta ter sido feita depois da medição manual, podendo considerar que o averiguador possuía mais prática, ou devido ao fato de que no computador a imagem é maior (mesmo não sendo usado o zoom) e, não tendo o papel Ultraphan na frente da foto, a marcação dos pontos se torna mais nítida e mais fácil.

Mas estas diferenças entre as médias foram bem pequenas, então pode-se considerar as médias de ambos os métodos de medição confiáveis, com uma maior segurança para as médias por computador.

O trabalho de Brangeli et al. ${ }^{3}$ constatou que para a maior parte das medidas (quase a totalidade) não há diferença estatisticamente significante entre a medição manual e por computador.

Já Martins et al. ${ }^{9}$, com dois operadores, testaram os métodos manual e o computadorizado. Encontraram erros significantes na repetição de mensurações cefalométricas em ambos os métodos, bem como os erros casuais que foram semelhantes nos dois métodos. Entretanto, El-Mangoury et al. ${ }^{7}$, que mediram fotografias de perfil manualmente e depois mediram estas fotografias já marcadas por meio do computador, não encontraram diferenças estatisticamente significante entre os dois métodos, mostrando que as diferenças acontecem na marcação dos pontos e não na diferença metodológica (manual x computador).

A análise do perfil facial em fotografias padronizadas está indicada na documentação ortodôntica, pois esta é fácil de ser obtida, de baixo custo e é um auxiliar importante para o diagnóstico e para o planejamento ortodôntico e/ou orto-cirúrgico, e também para a comunicação com o paciente.

A realização desta análise estaria indicada tanto através da medição manual como por computador. Tendo em vista que a utilização do software para estas medições demora aproximadamente apenas 15 minutos, contra uma hora ou mais, necessária para a medição manual, sugere-se a utilização do software para a execução destas medidas, visto que a presença de computadores na clínica ortodôntica hoje em dia é uma realidade.

Sugere-se os valores apresentados nas tabelas 1, 2 e 3 como padrão para adultos jovens do gênero masculino, para as medidas realizadas manualmente ou por computador em fotografias padronizadas.

\section{CONCLUSÕES}

Em vista dos resultados expostos e discutidos, pode-se concluir que:

a) Considerando que houve diferença entre as medições manual e por computador, deve-se utilizar as médias respectivas para cada tipo de medição.

b) Os dois tipos de medição são confiáveis, sendo a medição por computador mais precisa, pois somente uma medida mostrou diferença significativa entre a primeira e a segunda medição. Além disso, devido à praticidade e agilidade da medição por computador, esta seria a mais indicada. 


\title{
Facial profile analysis of esthetically harmonious young male adults in standardized photographs: comparison of manual versus computerized measurements
}

\begin{abstract}
Aim: To analyze the young male adult facial profile using standardized photographs and compare manual measuring procedures to the measuring procedures with the software Cef X (CDT). Methods: The sample consisted of 40 Brazilian Caucasian individuals, with an average age of 21.9 years, who possessed pleasant profiles, normal occlusion, and no history of previous orthodontic treatment. Colored photographs measuring $10 \mathrm{~cm} \times 15 \mathrm{~cm}$ were taken in a standard way with the subject in natural head position. The photographs were measured manually, using dial caliper and a protractor, as well as using the Cef $X(C D T)$ computer program. Thirty six facial variables were analyzed with linear, angular, and proportional measurements. Results: Manual and computerized measurement scores presented different statistics to 24 variables. In the computerized method only one measurement showed significant difference between the first and the second measuring whereas in the manual method eight measurements presented differences. Conclusion: Considering that there were differences in manual and computerized measurements, one must use respective mean values for each type of measuring procedure. Both types of measurement are reliable although the computerized one is more accurate. Due to the convenience and swiftness of the computerized measuring, it is best recommended.
\end{abstract}

Key words: Facial analysis. Facial profile. Orthodontics. Standardized photographs.

\section{REFERÊNCIAS}

1. ARNETT, G. W.; BERGMAN, R. T. Facial keys to orthodontic diagnosis and treatment planning (Part I). Am. J. Orthod. Dentofacial Orthop., St. Louis, v. 103, no. 4, p. 299-312, Apr. 1993.

2. BISHARA, S. E. et al. A computer assisted photogrammetric analysis of soft tissue changes after orthodontic treatment. Part I: methodology and reliability. Am. J. Orthod. Dentofacial Orthop., St. Louis, v. 107, no. 6, p. 633-639, June 1995.

3. BRANGELI, L. A. M. et al. Estudo comparativo da análise cefalométrica pelo método manual e computadorizado. Rev. Assoc. Paul. Cir. Dent., São Paulo, v. 54, n. 3, p. 232-241, maio/jun. 2000.

4. CAMPOS, H. Estatística experimental não-paramétrica. 4. ed. Piracicaba: Fealq, 1983.

5. CHIU, C. S. W.; CLARK, R. K. F. Reproducibility of natural head position. J. Dent., Guildford, v. 19, no. 2, p. 130-131, Apr. 1991.

6. CUMMINS, D. M.; BISHARA, S. E.; JAKOBSEN, J. R. A computer assisted photogrammetric analysis of soft tissue changes after orthodontic treatment. Part II: results. Am. J. Orthod. Dentofacial Orthop., St. Louis, v. 108, no. 1, p. 3847, July 1995.

7. El-MANGOURY, N. H. et al. Faciometrics: a new syntax for facial feature analysis. Int. J. Adult Orthodon. Orthognath. Surg., Chicago, v. 11, no. 1, p. 71-82, 1996.

8. HOUSTON, W. J. B. The analysis of errors in orthodontic measurements. Am. J. Orthod., St. Louis, v. 83, no. 5, p. 382-390, May 1983

9. MARTINS, L. P. et al. Erro de reprodutibilidade das medidas cefalométricas das análises de Steiner e de Ricketts, pelo método convencional e pelo método computadorizado. Ortodontia, São Paulo, v. 28, n. 21, p. 4-17, jan./abr. 1995.
10. RECHE, R. et al. Análise do perfil facial em fotografias padronizadas. Rev. Dental Press Ortodon. Ortop. Facial, Maringá, v. 7, n. 1, p. 37-45, jan./fev. 2002.

11. REIS, S. A. B. et al. Análise facial subjetiva. Rev. Dental Press Ortodon. Ortop. Facial, Maringá, v. 11, n. 5, p. 159-172, set./ out. 2006.

12. REIS, S. A. B. et al. Pergunte a um expert - Parte I. Rev. Clin. Ortodon. Dental Press, Maringá, v. 6, n. 4, p. 15-24, ago./set. 2007.

13. STRAUSS, R. A. et al. Variability of facial photographs for use in treatment planning for Orthodontics and Orthognathic Surgery. Int. J. Adult Orthodon. Orthognath. Surg., Chicago, v. 12 no. 3, p. 197-203, 1997.

14. SUGUinO, R. et al. Análise facial. Rev. Dental Press Ortodon. Ortop. Facial, Maringá, v. 1, n. 1, p. 86-107, set./out. 1996.

15. VERONA, J. et al. Análise facial frontal masculina em repouso e durante o sorriso, métodos manual e computadorizado, em fotografias padronizadas. Parte I. J. Bras. Ortodon. Ortop. Facial, Curitiba, v. 11, n. 64, p. 379-394, 2006.

16. VERONA, J. et al. Análise facial frontal masculina em repouso e durante o sorriso, métodos manual e computadorizado, em fotografias padronizadas. Parte II - Análise do sorriso. J. Bras. Ortodon. Ortop. Facial, Curitiba, v. 12, p. 560-575, 2007.

17. VIAZIS, A. D. A new measurement of profile esthetics. J. Clin. Orthod., Boulder, v. 25, no. 1, p. 15-20, Jan. 1991.

18. ZAR, J. H. Biostatistical analysis. 3rd ed. New Jersey: Prentice Hall, 1996.
Endereço para correspondência

Isabella Cabral Alexandre Schlickmann

Av. Hercílio Luz, 839 - apto. 902

CEP: 88.020-001 - Florianópolis /SC

E-mail: icasbn@hotmail.com 